\title{
Traditional minicomputers: A continuing role in the laboratory
}

\author{
HOWARD L. KAPLAN \\ Addiction Research Foundation, Toronto, Ontario MSS 2S1, Canada
}

\begin{abstract}
The traditional laboratory minicomputer was programmed in assembler, in a home-built language, or in a fast, compiled language with access to assembler-coded subroutines. This software strategy remains useful on inexpensive, microcomputer-based hardware for many applications in which the computer must respond to external events within a few milliseconds. The benefits of rapid response and sometimes lower hardware expense may require the cost of increased software development complexity and time.
\end{abstract}

With the rapidly declining cost of microcomputers, it is reasonable to ask what role, if any, the traditional minicomputer can play in the psychology laboratory. To answer this question, we must first understand what is meant by a minicomputer and then ask whether any of those aspects of its definition are critical to its effective use in the control and analysis of experiments. What I will argue is that the hardware differences between minicomputers and microcomputers are generally not important but that the traditional minicomputer software approach handles many problems that the usual microcomputer software approach cannot effectively handle.

What I mean by a traditional minicomputer is not only a question of the hardware technology used to build the computer or a simple matter of size, with minicomputers being larger than microcomputers. It is also not simply a matter of vendor, with a minicomputer being purchased from Digital Equipment Corporation, Data General, Sperry-Univac, HewlettPackard, or a similar manufacturer of many years' experience, with a list price, weight, and complexity high enough to warrant on-site company service. What is more important than the traditional product of the vendor, and what I make the basis of my definition of a minicomputer, is the ease with which very specialized software can be installed on the system. Many of today's microcomputers, especially the lower cost ones designed for home and hobby use, contain BASIC in read-only memory (ROM), and their resources are optimized for programming in BASIC. It is difficult or impossible to regain those resources (especially memory space) for other languages or for modification of the BASIC. What there is of an operating system is also in ROM, allowing very little opportunity for the user to modify it. In contrast, a minicomputer usually has at most a mass-storage bootstrap program in ROM, leaving the rest of the memory space to be implemented in randomaccess, read-write memory that can contain straight vendor-supplied programs, home-compiled programs, or third-party software for special applications. For users with very specialized applications, such freedom to use the hardware in its least constrained mode may be critical. Under this definition of a minicomputer, most S-100 microcomputer systems with very limited ROM should be classed with the minicomputers, despite their single-chip CPUs and low cost. Even a product such as the Rockwell AIM-65, which costs about $\$ 700$ and has a machine language monitor in ROM, is closer in philosophy to the PDP-8 of 10 years ago than to the Apple or Commodore PET of today, and it should appeal to the experimenter whose needs are for a minicomputer, as I have defined one. At the other end of the cost spectrum, when a computer in the $\$ 100,000$ range is dedicated to a single experiment at a time, then it is being used like a minicomputer, rather than like a miniature timesharing system.

The resource for which the minicomputer is the best solution, not because of the hardware but because of the software, is effective processing speed. Typical minicomputer applications are coded in a mixture of assembler, FORTRAN or PASCAL, and home-built languages. Because these languages are not generally restricted to floating-point data representations, are precompiled rather than interpreted, and provide for reasonably complex subroutine calling sequences, they can execute hundreds of times faster than interpreted languages such as BASIC. This speed makes it practical to control events with a grain measured in milliseconds and to make decisions based on external events in similarly short intervals. It should be obvious that many experiments in perception and sensation, especially those using a computer to emulate a high-speed tachistoscope, require such speed.

What may not be as obvious is that even single-box operant paradigms also require a large number of decisions to be made in a short time. When the input and output events are coded as single bits within input or output words, and when the task of deciding which events have occurred and how to process them involves combinations of arithmetic operations designed to simulate simple masking operations, delays on the order 
of $10 \mathrm{~s}$ of milliseconds may be created in responding to barpresses, keypecks, and similar events.

As an example, consider eight response keys wired to an 8-bit input port. To test for the binary value 4 bit in most versions of BASIC, the programmer must code something like this.

\section{B=INP(61): REM READ THE PORT}

\section{B4=INT((256+B)/4): REM FORCE B POSITIVE AND DELETE 2 BITS}

\section{IF(B4=2*INT(B4/2))THEN 250: REM B4 WAS EVEN}

This ridiculous segment of code requires an addition, two divisions, one multiplication, two function calls, and one subtraction or comparison, all in floating point, to test a single bit. The PET and the Compucolor II, two typical microcomputer implementations of BASIC that perform all computations in floating point, require $27 \mathrm{msec}$ each for this code. Versions of BASIC that can store values as integers, to save memory, but reconvert to floating point for all arithmetic will take even longer. The incrementally compiled BASIC on the Hewlett-Packard System 9845A, in which the I/O ROM provides a BINAND function for bit tests like these, requires $4 \mathrm{msec}$ as coded above and $1.7 \mathrm{msec}$ using the special function. In contrast, a two-parameter function call in a well designed 8080 FORTRAN would take on the order of 50 memory cycles. In 8080 assembler, an optimized routine to perform the test takes about half as long. If the bit to be tested does not vary, a Z-80 can perform the test in one machine instruction. This range of four orders of magnitude to perform the same function strongly suggests that the software at the slow end of the range is not suitable for the task at hand.

Delays of $30 \mathrm{msec}$ may be barely tolerable for a single box, but the possibility of cascading such delays makes multiple-box control from BASIC highly inadvisable. A more appropriate language for such operant control is a state-diagram language such as ACT, SCAT, or SKED, which has traditionally been available on minicomputer hardware. There is nothing inherent in microcomputer hardware that prevents such a language's being implemented on it, and such a solution makes economic sense (Butler, 1980). What we are likely to find, though, is that when such an implementation uses an off-the-shelf hardware package, it bypasses many of the features of the system software, replacing it with a very specialized operating system suited for the kinds of interrupts and events peculiar to operant control.

The reason that languages such as BASIC are often ill suited for specialized control is that every computer language is designed to implement a particular model of what is important in the computing universe. FORTRAN, for example, is optimized for efficient scientific calculations, at the cost of less than ideal debugging facilities, character manipulations, and program structure. BASIC sacrifices much in the way of execution efficiency for ease in programming and debugging of arithmetic and string operations. Assembler languages sacrifice almost everything else for extremely detailed control over computer operations. When the model of what is important in computing is shared by the experimenter and by the vendor or language developer, then a largely prepackaged solution may be appropriate. When the models are quite different, then the wrong prepackaged solution may be worse than starting with almost no software at all.

Some of Shannon's early work in information theory showed that it is possible to improve coding efficiency by making the frequently used messages correspond to short code sequences, even if that means increasing the length of code required to transmit uncommon messages (Singh, 1966). That finding is relevant when we consider that a program is a message, written in a very special code, from a programmer to a computer. That code has been designed to make some messages particularly easy to transmit, expressible in short sequences of characters that (we hope) result in a small number of machine operations at execution time. Other messages that the language designer expected to be infrequently sent may require many characters to express and result in many cycles of machine time to execute. It is in these cases, when the messages we wish to send to our computers can be expressed poorly, unnaturally, or inefficiently in the codes of languages normally supplied with the computers, that we need to consider bypassing the attractive built-in operating systems of many microcomputers and, instead, coping with the more difficult but more versatile code sequences necessary to communicate with a minicomputer.

An operating system is the system software that controls the allocation of computer resources among competing tasks and that is essentially reused even though the application tasks change. On a large timesharing or batch-processing system, the resources most obviously controlled are the mass storage, input, and output peripherals: terminals, printers, disks, and tapes. In a laboratory environment, the resources that most obviously need control are the CPU time and the available I/O channels. The times when control must be switched or conflicts resolved are generally signaled by external interrupts, and so much of the role of the operating system can be described as interrupt handling. If critical resources are not available upon interrupts, or if it cannot be predicted whether or not they will be available, real-time laboratory management becomes impossible.

The operating systems supplied with most hobbygrade computers are fairly primitive and cannot handle interrupts other than the keyboard and a line-frequency clock. Even these interrupts allow only for the updating of an unprocessed-character buffer or a software clock, with immediate return to the interrupted program. Such 
operating systems can cope only with experiments in which the important events occur in a predictable, strict sequence. Such experiments include many cognitive studies, in which each stimulus sequence is followed by one response or by a list of responses in sequence. If the computer is fast enough, then operant paradigms can also be considered as strict sequences, in which each 16-msec clock tick can be treated as a stimulus that is followed by a response of either barpress or bar-ignore. Such an approach requires that no clock value or response trigger any processing or $I / O$ that extends into the next clock period. In most cases, this means that all relevant data must be saved in memory until a session ends, because the operating system cannot simultaneously manage experimental control and disk, tape, or hard-copy output. In summary, the experiments least suitable for implementation under typical off-the-shelf operating systems are those requiring nontrivial multiprogramming techniques (Kaplan, 1980). While such multiprogramming real-time systems can be home-built and while some are now being advertised for low-cost microcomputers, the best known examples are still those from traditional vendors, such as RT-11 and RSX-11 from Digital Equipment, VORTEX from Sperry-Univac, RTE from Hewlett-Packard, and RDOS from Data General.

Another resource that distinguishes many minicomputers from microcomputers is the sophistication of their direct memory-access, or DMA, systems, needed in the control of synchronous devices during real-time operations. A synchronous device is one that requires very closely controlled time intervals between successive input or output operations. Tapes and disks are typical synchronous mass storage devices, whereas digital-toanalog and analog-to-digital converters must be used in a synchronous mode for waveform synthesis and analysis. Without DMA, the computer cannot maintain control of a synchronous device except when it is dedicated to that device. With DMA, however, separate hardware maintains the timing accuracy of transfers between memory and an external device, and the computer can busy itself processing irregular interrupts or performing less time-critical calculations. DMA capability of some sort is needed for logging results to disk without interrupting the flow of a long experiment, and multiple-channel capacity is needed for logging results when recording or generating analog waveforms while such disk transfers are occurring. While some DMA controllers are now being made available for microcomputer systems, it is still in the minicomputers that the more sophisticated, multiplechannel (or multiple-port) capability is found. Further, some microcomputer DMA circuitry totally inhibits the CPU during data transfers, rather than sharing memory cycles with the CPU. While this allows inter- facing of devices that are faster than the CPU can control, it prevents overlapping $\mathrm{I} / \mathrm{O}$ operations with the CPU's exercise of other control functions. This is not a matter of software versatility, as some of the other considerations are, but it is one of the few meaningful hardware differences between the ways minicomputers and microcomputers have traditionally been designed.

In summary, a minicomputer approach is suitable for the experimenter who is capable of learning about some of the inner workings of the computer system and does not need to treat it as a black box that speaks only BASIC. The experimenter will need to learn the computer's assembler language for occasional tasks, even if most day-to-day work is done in FORTRAN or BASIC, will need to read manuals that discuss interrupts, vectors, CPU cycles, and memory maps, and will eventually write programs that are less portable than might otherwise be wished. Despite some manufacturers' attempts to advertise differences among their machines, many microcomputers are designed to obscure hardware differences and present the user with a surface structure that differs very little from one computer to another. In contrast, the kind of code allowed by a minicomputer or assembler-programmed computer may be the kind of code that makes maximum use of some of the peculiar resources that distinguish one computer from another and is difficult to share with users of different hardware.

Some individuals may interpret this as suggesting that they purchase raw computers with little or no software, so that they can experience the joy of building operating systems from scratch. That is not what I am advocating. Instead, I am advocating that they analyze the demands they are willing to make upon their computers and themselves. For relatively nondemanding applications and for experimenters who are not interested in building tools, a relatively slow but friendly computer may be more than adequate for their experiments, especially if experimental events happen over large fractions of a second rather than over milliseconds. For those other experimenters willing to develop some expertise in computing itself, in addition to expertise in psychology, the potential power of software that is less idiot-proof than BASIC may be the only appropriate solution to their laboratories' needs.

\section{REFERENCES}

Butle R, A. F. MicroSKED. Behavior Research Methods \& Instrumentation, 1980, 12, 152-154.

KAPLAN, H. L. Applications of multiprogramming to real-time experiments in psychology. Behavior Research Methods \& Instrumentation, 1980, 12, 137-151.

Singh, J. Great ideas in information theory, language, and cybernetics. New York: Dover, 1966. 